\title{
Utilisation du test ELISA de détection des antigènes circulants de trypanosomes dans le cadre d'un suivi épidémiologique dans la zone de Sidéradougou, Burkina Faso
}

\section{Mots-clés}

Bovin - Trypanosomose - Test ELISA Antigène - Trypanosoma - Prélèvement sanguin - Diagnostic - Infestation Epidémiologie - Burkina Faso.

\author{
A. Delafosse ${ }^{1}$ Z. Bengaly ${ }^{1}$ G. Duvallet 2
}

\section{- INTRODUCTION}

La trypanosomose animale africaine (TAA) est un facteur limitant du développement de l'élevage dans les zones subhumides d'Afrique. Différentes techniques permettent de diagnostiquer cette parasitose, la plus usuelle étant l'examen microscopique direct de l'interface entre les globules rouges et le plasma ou buffy coat après une centrifugation en tube capillaire (8).

Ce diagnostic, par mise en évidence directe des trypanosomes, a l'avantage d'être très spécifique. Son inconvénient réside dans sa mauvaise sensibilité, estimée expérimentalement à 55 p. 100 pour les infections à Irypanosoma congolense et à 48 p. 100 pour les infections à $T$. vivax (5). Le diagnostic parasitologique sous-estime donc l'incidence de la TAA.

Pour tenter d'améliorer le dépistage des animaux infectés, un test de délection des antigènes circulants de trypanosomes, de type ELISA, a été proposé en 1989 par Nantulya et Lindqvist (9). Ce test utilise des anticorps monoclonaux spécifiques dirigés contre T. congolense, $T$. vivax et T. brucei. Masake et Nantulya (6) ont

1. CIRDES, 01 BP 454, Bobo-Dioulasso 01, Burkina Faso

2. CIRAD-EMVT, Campus international de Baillarguet, BP 5035,

34032 Montpellier, France estimé la sensibilité de ce test, lors d'infections expérimentales par T. congolense, à 94,3 p. 100 chez les chèvres et 82,5 p. 100 chez les bovins.

Une évaluation menée au Centre international de RechercheDéveloppement sur l'Elevage en Zone subhumide (5) a permis, à partir d'infections expérimentales chez des petits ruminants, de calculer des sensibilités de 63,2 p. 100 lors d'infections à $T$. congolense et de seulement 9,9 p. 100 lors d'infections à T. vivax.

La spécificité de ce test a été évaluée à partir de plasmas provenant de régions indemnes de trypanosomes pathogènes. Ainsi, sur 40 plasmas de bovins prélevés en Europe et testés vis-à-vis de trois antigènes monoclonaux spécifiques, il n'y a eu aucun faux positif (5).

L'existence d'éventuelles réactions croisées entre les différentes espèces de trypanosomes n'a jamais été évaluée expérimentalement. Dans des conditions naturelles, seule l'absence d'interaction des infections à $T$. theileri avec le diagnostic des trypanosomoses animales par détection des antigènes circulants, a pu être montrée (3).

Ce travail cherche à évaluer l'intérêt du test de détection des antigènes circulants dans le cadre d'un suivi épiđémiologique de ter- 
rain. Pour cela, des plasmas provenant d'une cohorte de bovins suivis pendant un an, de juin 1986 à juin 1987, ont ćtć analysés a posteriori en 1993. Ces bovins proviennent en partie de quatre villages situés dans la zone pastorale de Sidéradougou (Burkina Faso), région indemne de glossines à la suite d'un vaste programme d'éradication par lâchers de mâles stériles $(1,2)$.

\section{MATERIEL ET METHODES}

La population éludiée est une cohorte de 216 bovins répartis dans quatre villages situés dans la zone indemne de glossines. Deux villages, Sidéradougou et Dingasso, sont en bordure de cette zone, alors que les deux autres, Moussobadougou et Nyarafo, sont enclavés (tableau I).

La population témoin est constituée d'une cohorte de 81 bovins provenant d'un village (Kassandé) situé à $20 \mathrm{~km}$ au sud de la zone libérée de glossines.

\section{TABleau I}

\section{Caractéristiques de la population étudiêe}

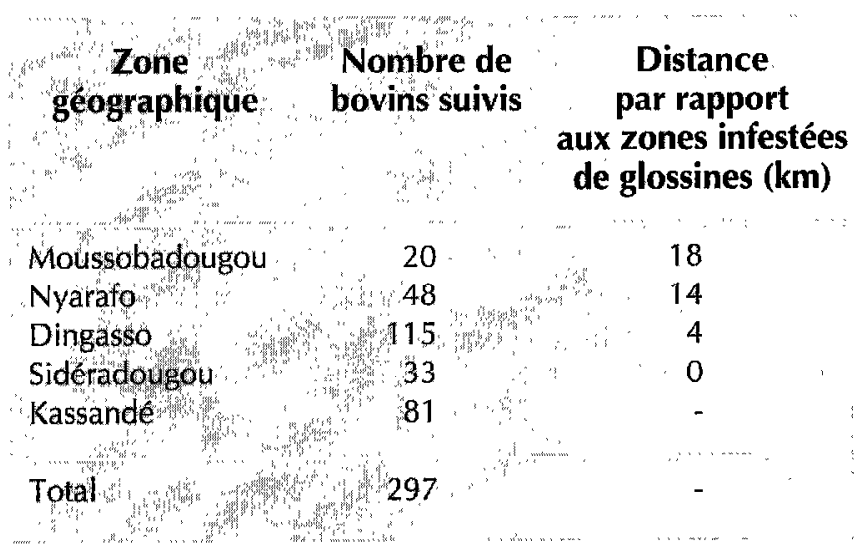

Les animaux ont été suivis pendant une année, de juin 1986 à juin 1987. Des prélèvements de sang ont été effectués en saison des pluies (septembre 86), en début de saison sèche (janvier 87) et en fin de saison sèche (mai 87).

Sur chaque prélèvement, un diagnostic parasitologique a été effectué immédiatement après la prise de sang. La technique utilisée était l'examen direct du buffy coat après une simple centrifugation en tube capillaire (8). Les bovins positifs étaient traités avec de l'acéturate de diminazène (Berenilß) à la dose de $7 \mathrm{mg} / \mathrm{kg}(1,7)$.

Après sédimentation des hématies, les plasmas étaient prélevés et une goutte de benzoate de sodium était ajoutée afin d'améliorer leur conservation. Les plasmas ont ensuite été congelés et conservés à $-20^{\circ} \mathrm{C}$ jusqu'au mois d'août 1993 . A ce moment-là, les échantillons ont été décongelés et testés avec la technique ELISA de détection des antigènes circulants de trypanosomes (9).

Par ailleurs, les plasmas provenant de 23 bovins infectés par $T$. congolense (10 plasmas) ou $T$. vivax (13 plasmas) et prélevés en 1986 dans un village proche de Bobo-Dioulasso (Satiri) ont également été testés, afin d'évaluer l'état de conservation des antigènes circulants.

Les seuils de positivité retenus étaient une densité optique (D.O.) de 0,05 pour le diagnostic des infections à $T$. congolense et à $T$. brucei, et une D.O. de 0,02 pour celles à $T$. vivax. Ce protocole a été élaboré à la suite de l'évaluation faite au CIRDES (5).

La saisie et l'analyse des données ont été effectuées avec le logiciel EPI INFO.

\section{RESULTATS}

Les sensibilités du test ELISA pour le diagnostic des infections à $T$. congolense et à $T$. vivax ont été calculées à partir des plasmas des bovins parasitologiquement positifs (tableau II). Sur 50 plasmas, 23 provenaient de Satiri, 10 de Kassandé (zone témoin) et 17 de la zone pastorale de Sidéradougou.

\section{TABLEAU II}

\section{Résultats du test de détection des antigènes circulants chez des bovins infectés par $T$. congolense et $T$. vivax}

$\begin{array}{lccc}\begin{array}{l}\text { Test ELISA } \\ \text { (anticorps } \\ \text { monoctonal } \\ \text { specifique) }\end{array} & \begin{array}{c}\text { Bovins infectés } \\ \text { par T. congolense }\end{array} \\ \text { par } T \text {. vivax } \\ \text { positif" }\end{array}$

La sensibilité d'un test (Se) se définit comme le rapport des vrais positifs sur la somme des vrais positifs et des faux négatifs.

On a donc ici

pour $T$. congolense, $\mathrm{Se}=6 / 10=0,6$

et pour $T$, vivax, $\mathrm{Se}=9 / 40=0,22$.

Les résultats des suivis parasitologique et sérologique sont exprimés en fonction du lieu et de la date des prélèvements (tableaux III et IV).

TABLEAu III

Résultats du suivi parasitologique

Nombre de bovins positifs à Y'examen parasitologique

Total sept. 86 jan. 87 mai 87

$\begin{array}{lcccc}\text { Moussobadougou } & 0 & 0 & 0 & 0 \\ \text { Nyarafo } & 0 & 0 & 1 & 1 \\ \text { Dingasso } & 0 & 12 & 3 & 15 \\ \text { Sidéradougou } & 0 & 0 & 1 & 1 \\ \text { Kassandé } & 0 & 1 & 9 & 14 \\ \text { Total } & 0 & 13 & 14 & 27\end{array}$

$\mathrm{Rq}$ : tous les trypanosomes isolés appartenaient à l'espèce $T$. vivax. 
TABLEAU IV

Résultats du suivi sérologique

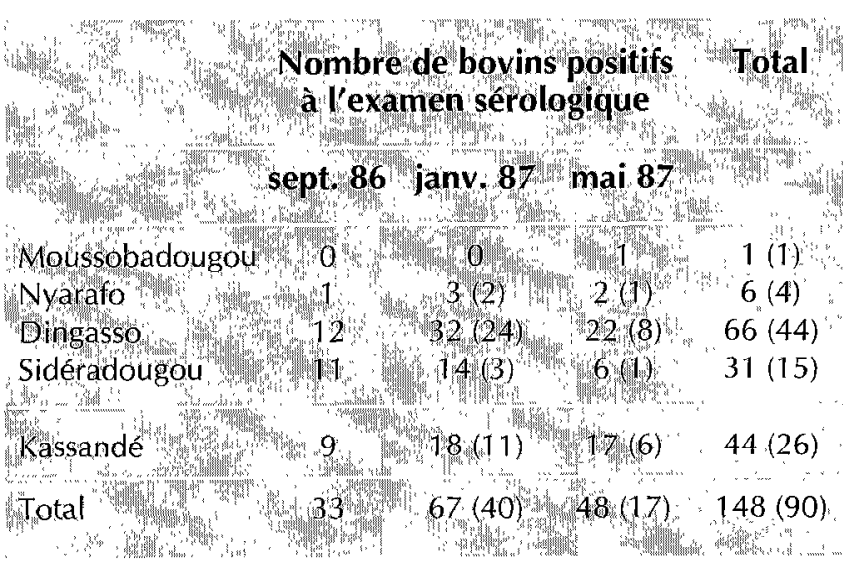

Les thiflres entre parenthèses représentent les nouveaux cas.

A partir de ces résultats, des taux d'incidence annuels de la TAA ont été calculés. Les taux d'incidence (TI) calculés avec les résultats du diagnostic parasitologique seront appelés par la suite taux. d'incidence parasitologiques, ceux calculés avec le test ELISA seront appelés taux d'incidence sérologiques.

Un taux d'incidence est défini comme le rapport du nombre d'individus tombés malades au cours d'une période donnée, sur le nombre d'individus-temps exposés au risque pendant la même période. Pour simplifier les calculs, on a considéré que les bovins positifs s'étaient infectés au milieu de la période séparant deux prélèvements, soit deux mois avant le diagnostic de la TAA. Les bovins traités à l'acéturate de diminazènc ont été considérés comme indemnes de trypanosomes et donc de nouveau exposés au risque d'infection. Les animaux positifs au test ELISA et non traités ont été considérés comme infectés et donc non exposés au risque d'infection.

Les taux d'incidence annuels de la TAA ont été calculés pour chaque zone retenue dans l'étude (tableau V).

\section{TABLEAU $V$}

\section{Taux d'incidence parasitologiques et sérologiques en fonction de la zone géographique}

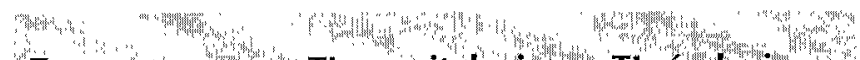
Zone Tharasitolngiques TI sérologiques

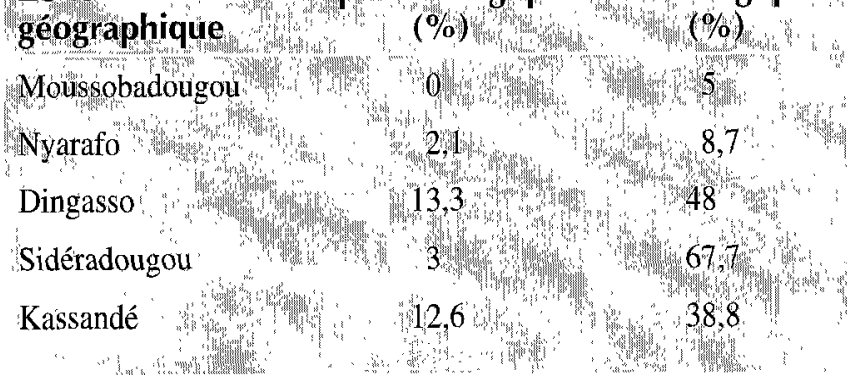

A titre d'exemple, le calcul des taux d'incidence à Dingasso est détaillé ci-après :

- Pour le TI parasitologique, le nombre de malades est de 15. Le nombre d'individus-temps (ici des bovins-mois) exposés au risque est de $(115 \times 4)$ bovins-mois du ler juin 86 au ler octobre 86 , de $[(12 \times 2)+(103 \times 4)]$ bovins-mois du ler octobre 86 au 1er février 87 , et de $[(3 \times 2)+(112 \times 4)]$ bovins-mois du 1er février 87 au 1er juin 87 . Ce qui fait cn tout 1350 boyins-mois exposés au risque.

Le taux d'incidence parasitologique annuel est alors de :

TIp $=(15 / 1350) \times 12=0,133$ soit 13,3 p. 100 .

- Pour le taux d'incidence sérologique, le calcul est le même mais les animaux positifs au test ELISA et non traités doivent être exclus du calcul du dénominateur car ils ne sont plus exposés au risque.

$\mathrm{TIs}=(44 /[(12 \times 2)+(103 \times 4)+(79 \times 4)+(24 \times 2)+(71 \times 4)+$ $(8 \times 2)]) \times 12=0,48$ soit 48 p. 100.

La figure 1 permet de comparer les taux d'incidence annuels de la TAA en fonction du type de diagnostic utilisé et du lieu de prélèvement.

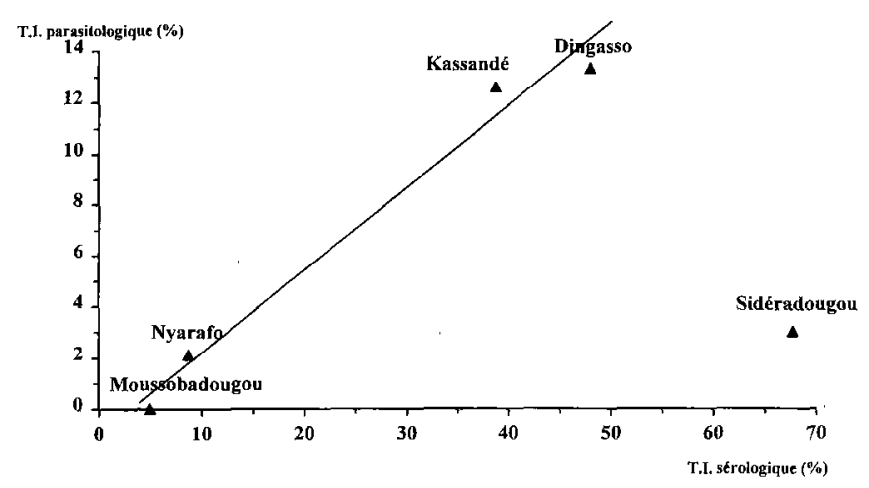

Figure 1 : résultats comparés des diagnostics parasitologique et sérologique.

Les taux d'incidence calculés avec le test ELISA diminuent lorsque la distance séparant le lieu de prélèvement et la limite de la zone traitée augmente (tableau VI, figure 2).

\section{TABLEAU VI}

TI calculés avec le test ELISA el dislances séparant le lieu de prélèvement et la limite de la zone traitée

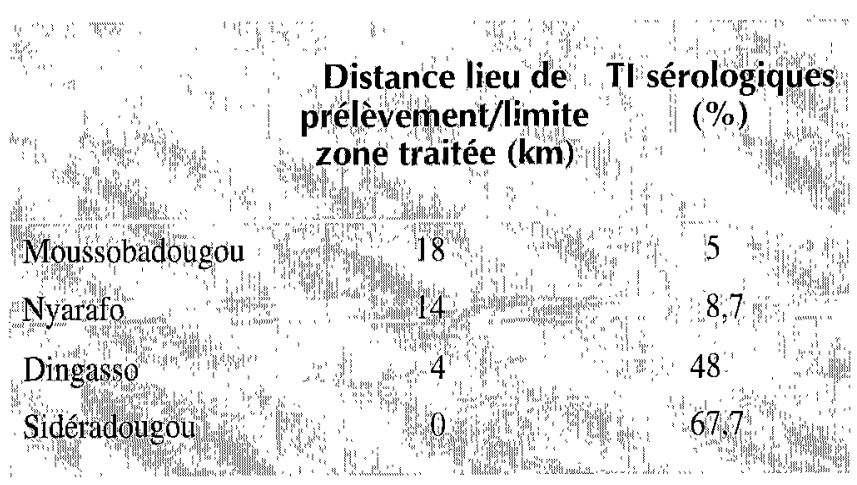




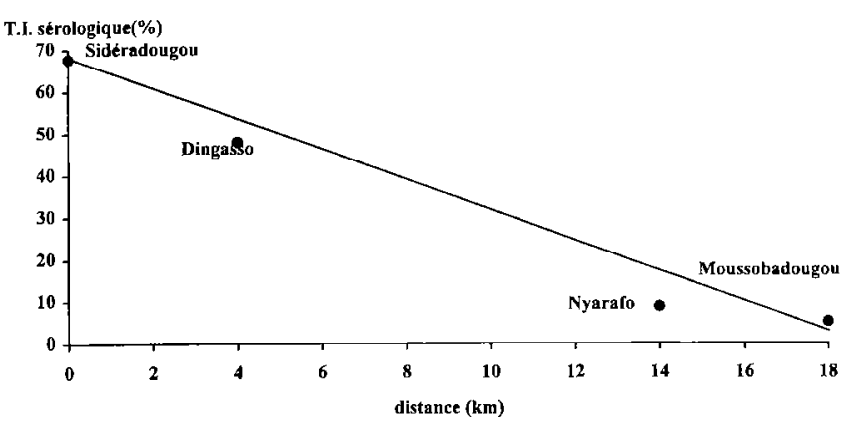

Figure 2 : répartition des $T I$ calculés avec le test ELISA en fonction de la distance séparant le lieu de prélèvement et la limite de la zone traitée.

\section{- DISCUSSION}

Les sensibilités du test de détection des antigènes circulants calculées dans cette étude sont proches de celles trouvées au CIRDES (5) lors d'infections naturelles chez des bovins. Il semble donc que les sept années de stockage des plasmas n'aient pas altéré les antigènes circulants. En effet, si cela avait été le cas, le grand nombre de faux négatifs aurait fait baisser les sensibilités du test. Ce test de détection des antigènes circulants a parfois montré une spécificité et une sensibilité réduites (4). Un nouveau protocole est en cours d'évaluation dans un projet coordonné par l'Agence internalionale pour l'Energie atomique (AIEA, Vienne, Autriche).

On note une bonne concordance entre les résultats des deux techniques de diagnostic, à l'exception de ceux obtenus dans le village de Sidéradougou.

A Moussobadougou et à Nyarafo, villages les plus éloignés des zones infestées de glossines, les taux d'incidence parasitologiques et sérolugiques sont faibles. Ainsi, à Moussobadougou, le TI parasitologique est nul alors que le TI sérologique est 8 fois plus faible que celui calculé dans la zone témoin. A Nyarafo, les TI parasitologique et sérologique sont respectivement 6 et 4,5 fois plus faibles que ceux obtenus à Kassandé (zone témoin). Les quelques cas observés pourraient être liés à la transmission mécanique de $T$. vivax par des diptères hématophages (tabanides, stomoxes) $(11,12)$.

A Dingasso, village situé en bordure de la zone traitée, des glossines avaient été capturées pendant le déroulement du suivi, marquant une tendance à la réinvasion de la zone $(1,7)$. Les TI parasitologique et sérologique calculés à Dingasso sont respectivement 1,05 et 1,25 fois plus élevés que ceux relevés à Kassandé.

A Sidéradougou par contre, les résultats sont divergents. Le TI parasitologique est 4 fois plus faible que celui de la zone témoin, alors que le TI sérologique est 1,75 fois plus élevé que celui calculé dans cette même zone. Ce phénomène pourrait s'expliquer par une sensibilité particulièrement faible du test parasitologique dans le village de Sidéradougou. En effet, le nombre de faux négatifs augmente si les bovins infectés contrôlent bien leur parasitémie, cc qui pcut ctre le cas s'ils se trouvent dans de bonnes conditions d'entretien (12). Dès lors, le nombre de trypanosomes par $\mathrm{ml}$ de sang est trop faible pour pouvoir être détecté après une simple centrifugation en tube capillaire (les seuils de détection étant de 500 trypanosomes $/ \mathrm{ml}$ pour T. brucei, de 1250 tryp./ml pour ' $I$.' vivax et de 6250 tryp./ml pour $T$. congolense) (10).

Ces résultats divergents pourraient également s'expliquer par un nombre important de faux positifs avec le test ELISA. Cela suppo- serait l'existence chez les bovins du village de Sidéradougou uniquement, d'un organisme entraînant des réactions croisées.

Une autre explication prend en compte les traitements trypanocides qui auraient été beaucoup plus nombreux autour de Sidéradougou. En effet, on y trouve de nombreux agents techniques et aussi le service provincial des ressources animales, ce qui faciliterait la réalisation de ces traitements en cas de maladies ou de suspicion par les éleveurs.

Par ailleurs, la corrélation observée entre le TI sérologique et la distance séparant le lieu de prélèvement de la limite de la zone infestée de glossines, laisse à penser que ce test permet de se faire une idée plus juste du contact entre les cheptels bovins et les vecteurs. Ainsi, le village de Sidéradougou étant situé en bordure de la zone infestée de glossines, il est probable que le cheptel suivi ait été cxposć au risque d'infection, ce qui apparaît uniquement avec le diagnostic sérologique.

Différents facteurs limitent les interprétations à donner à ces résultats. La bonne conservation des antigènes circulants n'a pu être démontrée formellement. Pour ce faire, il aurait fallu réaliser une série de tests avant la période de stockage puis comparer les résultats obtenus avant et après.

Par ailleurs, les cohortes suivies dans les différents sites retenus sont de tailles inégales. De plus, étant donné la mauvaise sensibilité du diagnostic parasitologique, il aurait été préférable de disposer d'effectifs plus importants. Ceci est particulièrement vrai dans les zones où la TAA a fortement régressé du fait de la disparition des vecteurs.

L'absence de données sur les modes de conduite des cheptels est également un handicap pour l'interprétation des résultats. Ainsi, dans le village de Sidéradougou, il aurait été important de connaître la localisation des zones de pâturages (en zone infestée ou en zone indemne de glossines)

Enfin, la distinction entre les différentes espèces de trypanosomes au nivcau du diagnostic sérologique n'a pas été prise en compte. En aucun cas, ce travail n'aborde le problème de la fiabilité du test de détection des antigènes circulants pour le diagnostic différentiel des infections à $T$. vivax, $T$. congolense ou $T$. brucei.

Ce travail présente deux originalités par rapport aux études épidémiologiques déjà effectuées sur le sujet.

Il s'agit tout d'abord d'une enquête sérologique effectuée a posteriori, sept années après la réalisation des prélèvements sur le terrain. Par ailleurs, la zone étudiée a été artificiellement libérée des glossines, les régions situées alentour restant infestées (2). A la suite du suivi parasitologique, la disparition de la trypanosomose avait été constatée, en dehors de réinfections localisées, liées à des réinvasions par les glossines (à Dingasso) $(1,7)$.

Les interprétations tirées de cette enquête sérologique permettent d'affiner ces résultats. On observe en effet des taux d'incidence croissants du centre de la zone pastorale (où ils sont faibles mais non nuls) vers sa périphérie où les TI sont équivalents à ceux calculés dans la zone témoin.

Le test ELISA de détection des antigènes circulants de trypanosomes pourrait être utilisé pour apprécier l'impact de la lutte antivectorielle sur l'incidence de la TAA.

Dans ce cadre, des cheptels sentinelles, situés dans les zoncs libćrées des glossines, pourraient être régulièrement prélevés afin de surveiller l'évolution des taux d'incidence. En cas de remontées significatives de ceux-ci, des sondages entomologiques confirmeraient ou non la réinvasion de la zone, ce qui déboucherait sur une nouvelle campagne de lutte. 


\section{Remerciements}

Nous tenons à remercier pour leur assistance le Dr Saydil M. Touré, Directeur Général du CIRDES, le Dr Bauer et l'équipe du laboratoire du CIRDES.

\section{BIBLIOGRAPHIE}

1. BAUER B., PETRICH-BAUER J., KABORE I, MATTAUSCH M., SOME J., TAMBOURA I., 1988. Epidemiological survey in the pastoral zone of Sidéradougou (Burkina Faso). Bobo-Dioulasso, Burkina Faso, CRTA/GTZ, 7 p. (rapport d'activité)

2. CUISANCE D., POLITZAR H., MEROT P., TAMBOURA I., 1984. Les lâchers de mâles irradiés dans la campagne de lutte intégrée contre les glossines dans la zone pastorale de Sidéradougou (Burkina Faso). Revue Elev. Méd. vét.Pays trop. 37, (4) : 449-467.

3. DELAFOSSE A., BENGALY Z., DUVALLET G., 1995. Absence d'interaction des infections à Trypanosoma theileri avec le diagnostic des try. panosomoses animales par détection des antigènes circulants. Revue Elev. Méd. vét. Pays trop. 48, (1) : 18-20

4. DESQUESNES M., de La ROQUE S., 1995. Comparaison de la sensibilité du test de Woo et d'un test de détection des antigènes de Trypanosoma vivax chez deux moutons expérimentalement infectés avec une souche guyanaise du parasite. Revue Elev. Méd. vét. Pays trop. 48, (3) : 247-253

5. KANWE A.B., BENGALY Z., SAULNIER D., DUVALLET G., 1992 Fvaluation du test de détection des antigènes circulants de trypanosomes à I'aide d'anticorps monoclonaux. Infections expérimentales et naturelles. Revue Elev. Méd. vét. Pays trop. 45, (3-4) : 265-271.
6. MASAKE R.A., NANTULYA V.M., 1991. Sensitivity of an antigen detection enzyme immunoassay for diagnosis of Trypanosoma congolense infections in goats and cattle. J. Parasitol. 7, (2) : 231-236.

7. MATTAUSCH M., 1990. Epidemiologische Untersuchungen des Trypanosomosis von Rind und Schaf im Anschlu8 an eine TsetsefliegenTilgung in des Weidegebieten von Sidéradougou, Burkina Faso. Hannover, Allemagne, Tierärtz. Hochsch. Dis., 134 p.

8. MURRAY M., MURRAY P.K., Mc INTYRE W.I.M., 1977. An improved parasitological technique for the diagnosis of African trypanosomiasis. Trans. R. Sor. trop. Med. Hyg. 71 : 325-326.

9. NANTULYA V.M., LINQVIST K.J., 1989. Antigen-detection enzyme immunoassays for the diagnosis of $T$. vivax, $T$. congolense and $T$. brucei infections in cattle. Trop. Med. Parasitol., 40: 267-272.

10. PARIS J., MURRAY M., MCODIMBA F., 1982. A comparative evaluation of the parasitological techniques currently available for the diagnosis of African trypanosomiasis in cattle. Acta Tropica, 39: 309-316.

II. SOLANO P., AMSLER-DELAFOSSE S., 1995. Irypanosoma congolense chez différentes espèces de taons (Diptera : Tabanidae) au Burkina Faso. Revue Elev. Méd. vét. Pays trop., 48 (2) : 145-146.

12. TRONCY P.M., ITARD J., MOREL P.C., 1981. Précis de parasitologie vétérinaire tropicale. Maisons-Alfort, France,IEMVT, $717 \mathrm{p}$.

Reçu le 16.1.96, accepté le 10.6 .96 


\section{Summary}

Delafosse A., Bengaly Z., Duvallet G. Use of Trypanosoma antigen detection ELISA during an epidemiological survey in the Sideradougou area, Burkina Faso

Plasmas taken during a parasitological survey on bovine trypanosomosis were tested a posteriori with an antigen detection ELISA test. The objective was to evaluate the value of this test for epidemiological monitoring. The results were compared to those obtained using the usual parasitological technique (buffy coat examination). Of the 297 zebu cattle heads sludied, 216 came from four villages in the pastoral zone of Sideradougou. A large eradication programme had led to the disappearance of tsetse in the area. Blood samples were taken during the rainy saison (September 1986), at the beginning (January 1987) and at the end (May 1987) of the dry season. The parasitological diagnosis was carried out in the field and plasmas were stored at $-20^{\circ} \mathrm{C}$. The serological lest was performed in August 1993. The incidence rates of bovine trypanosomosis obtained with antigen detection ELISA were low in the center of the zone. These rates decreased proportionally to the distance between the blood sampling area and the limit of the infested area. The results of the parasitological diagnosis were similar except for a village located on the border of the treated zone. The use of antigen detection ELISA confirmed the low incidence of trypanosomosis in the center of the area of Sideradougou following the eradication campaign. It also enables to obtain finer parasitological results pointing out the trypanosome persistence in peripheric zones, more exposed to reinfestations.

Key words: Cattle - Trypanosomosis - ELISA - Antigen Trypanosoma - Blood sampling - Diagnosis - Infestation Epidemiology - Burkina Faso.

\section{Resumen}

Delafosse A., Bengaly Z., Duvallet G. Uso del ELISA para la detección de antígenos circulantes de tripanosomas durante una encuesta epidemiológica en la zona de Sideradougou, Burkina Faso

En el transcurso de un seguimieto parasitológico de la tripanosomosis bovina, se obtuvieron plasmas, los cuales fueron examinados a posteriori, gracias al test de detección de antígenos circulantes. El objetivo era el de evaluar el interés de este test en la encuesta epidemiológica, comparando los resultados con los de un diagnóstico parasitológico común (examen del buffy coat). El estudio se realizó sobre una cohorte de 297 bovinos, de los cuales 216 provenían de cuatro pueblos de la zona pastoril de Sideradougnu, región indemne de glosinas, después de un amplio programa de erradicación. Las muestras de estos animales se obtuvieron durante la estación de las lluvias (septiembre 1986), así como al inicio (enero 1987) y al final de la estación seca (mayo 1987). El diagnóstico parasitológico se llevó a cabo en el campo y los plasmas se conservaron a $-20^{\circ} \mathrm{C}$. El diagnóstico serológico se realizó en agosto de 1993. Las tasas de incidencia de la tripanosomosis bovina, obtenidas mediante el test de detección de los antígenos circulantes, son bajas en los hatos localizados en el centro de la zona. Estas disminuyen proporcionalmente con la distancia entre el sitio de obtención de la muestra y el límite de la zona infestada. Los resultados del diagnóstico parasitológico concuerdan, excepto en el caso de un pueblo situado en la frontera de la zona estudiada. De esta manera, el uso del test de detección de antígenos circulantes, confirma la baja incidencia de la tripanosomosis en el centro de la zona de Sideradougou, después de la campaña de erradicación. Esto permite igualmente de definir los resultados de la encuesta parasitológica, mostrando la persistencia de los tripanosomas en las zonas periféricas, más expuestas a las reinfestaciones.

Palabras clave : Ganado bovino - Tripanosomosis - ELISA Antígeno - Tripanosoma - Muestro sanguineo - Diagnóstico' Infestación - Epidemiología - Burkina Faso. 